\title{
A SystemC cache simulator for a multiprocessor shared memory system
}

\author{
Alfred Mutanga \\ Institutional Planning and Quality Assurance, University of Venda, University Road, \\ Thohoyandou, Limpopo Province, 0950, South Africa
}

E-mail address: alfred.mutanga@univen.ac.za

\begin{abstract}
In this research we built a SystemC Level-1 data cache syste n a distrib 1 s ared memory architectural environment, with each processor having its own lo 1 cà Using a s $l$ of Fast-Fourier Transform and Random trace files we evaluated the cache perf nnce, on the number of cache hits/misses, of the caches using snooping and directory-ba ed cathe cohere protocols. A series of experiments were carried out, with the results of the ey eriments showing that the directory-based MOESI cache coherency protocol has a performance ge over the snooping Valid-Invalid cache coherency protocol.
\end{abstract}

Keywords: Cache Coherency; Cache Simulator;

\section{INTRODUCTION}

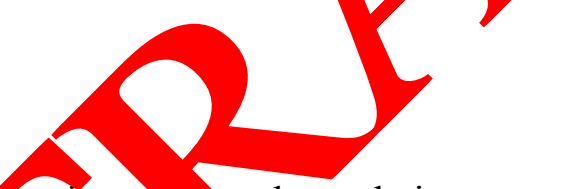

Architecturally, co puting sy ns have their memory organized hierarchically and this memory nomenclature is ntifically aned the memory hierarchy (Hennessey and Patterson, 2007; Stalling, 2012, The in the memory module is to the processor, the smaller and faster are the compon nts resulting 1 inverse relationship between the size and speed of the memory mod e. Ho ver, accsording to Hennessey and Patterson (2007) fast memory comes with cost imp ons as hese modules are relatively expensive per byte. Altogether the memorymodule a conputer system collectively allow the data and instructions to flow throy gh th ystem. central processing's unit registers are the most vital as these store the op ds a results of all computations capitalizing on the principle of locality (Hennessey and $\mathrm{Pa}_{\mathrm{a}}$ son, zov 1 ).

Th omputer program and data are typically stored on non-volatile storage such as disk drives and apes before execution but these are first loaded into main memory, which is much faster, but still significantly slower than the registers (Hennessey and Patterson, 2007, p. 288299). As an intermediate step in the memory hierarchy, caches were invented to avoid the penalties of memory access by keeping the most recently used data and delivery is much faster to the processor. Cache memories are therefore the conceptual foundation for this research. 


\section{PROBLEM STATEMENT}

As has been observed through various computer architecture research the problems facing the multicore processor systems at large are that, processor speeds are "rising dramatically at approximately $75 \%$ per year", according to McKee (2004). The memory clock speeds at the same time are increasing steadily at a paltry $7 \%$ per annum (Hennessey and Patterson, 2007). The research by NASA and scientists at the University of Virginia confirm this dilemma, that, there is a divergence in the operating speeds of memory architectures and processor systems, referred to as the Memory Wall (McKee, 2004). The challenge facing computer scienticts and engineers today is therefore to design a memory architecture that operates at th same $\mathrm{k}$ speeds as the processor architecture.

The computing industry facing the dilemma of the memory wall resolyed th $\mathrm{o}$ increa performance on computing systems should be as a result of build ig latench lera ce prefetching non-blocking cache memory systems (McKee, 2004).

This resulted in the computing industry building processor ch isting of larger cache memory systems and more latency tolerance on $\mathrm{cl}^{\mathrm{p}} \mathrm{p}$. Memo archi ectures are organized hierarchically, with the memory components near he process oeing smaller and faster (Hennessey and Patterson, 2007). Cache memar syste are the to prevent the penalties of memory access by keeping the most recent 1 or requentl, $d$ data and deliver it as fast as is possible to the processor. The memory $\mathrm{w} / 1$ results in memg ry being considered as the bottleneck for processor performance, and mod h computer Irchitectural designs feature different cache memory levels (Hennessey and Patte , 2007)

Caches exploit the benefits of temporal and spati. y of the data in the computer's main memory by having regular access patte mically each memory request goes through the cache memory and subsequently channell $d$ to an in or a higher level cache memory if the requested data or instruction is not found in nat caene.

Complications arise when mu e proce sors with each having a local cache have a shared main memory system he y cious caches keep private copies of shared data while being unaware of what is copies in the other caches, undefined cache performance behaviour y arise.

Cache coheren $\mathrm{pl}$ cols are ro aired to maintain the cache consistency of all the data stored in the diff loca ches (Leiserson and Mirmam, 2008). The cache coherency protocols consi of cache lin ate transitions that can be captured by cache simulators. However it not e to get the actual behaviour of these caches and also to prove the correctness of cache haviour. Despite their benefits, multiprocessors can only scale so far and eneck or when several CPUs on a board share a single memory system and a by (Hen issey an atterson, 2007).

th mearch we evaluated the performance of Level 1 data cache memory systems in a mult cessor environment by looking at the influence of the bus traffic, and cache coherence protocols, mer of processors and cache associativity. We addressed the following research questions:

1. To what extend do the number of processors in multiprocessor architectures affect the performance of Level 1 (L1) data cache memory systems?

2. How do cache coherency protocols influence the Levell data cache memory performances of multiprocessor architectures? 


\section{THEORETICAL FRAMEWORK}

The problems that have been identified for uniprocessors have been addressed by the development of multi-core architectures. The real world is parallel, and the reason why single processors have faced problems is that they have been executing instructions sequentially in short bursts of time. The real explanation why chip companies shift to multi-cores is prosaic in the sense that it includes several reasons that are not within the context of this research. There is an inherent concept that multi-cores increase the speeds of execution of multiple tasks, but achieving parallelism is not a trivial task (Nussbaum and Smith, 2002). What are the chollenges or problems which multi-core designers face? Let us look into these problems bri 1 y.

\section{1. Programmability}

Historically parallel processing computer architectures and mult cores have ated computer architecture designers and system software developers prog nin crall ng s. The programming challenges include intellectual programming skills dea develop programs for such systems, and the need for specialised software tools rogram $t$, the daunting task for programmers is on the "parallelisation of sequenti pro ms" (Szy Aowski, 2005). The multi-core programming model should be based sandar rogramming tools and programming languages. There are no real standards 1 the programm $s$ landscape of multicores (Duller and Towner, 2003; Towner et al., ? 04; Jourbet, 2068). Echoing the same sentiments about programming multi-cores (Leisers and Mirm m, 2008) wrote that "multicore processors are parallel computers and paralle mputer are notoriously difficult to program". Chris Jesshope identified 3 differ models ormunine/programming models which are sequential; ad-hoc parallel and fully para er 1 (Jesshope, 2008). Even though these programming models exist there is need to a $\mathrm{jr}$ ss thy issue of standards and automation of multi-core programming tasks (Bl 2009).

\section{2. Scalability}

Multi-cores reduc system la cy but one of the challenges that multi-core systems developers face is de elo systems at are scalable. Multi-cores produce tangible benefits but making the proce pa l brings with it programming challenges as mentioned before. Increasing more processor cor $/$ chip might entail that the whole system has to be rewritten (Blyler, 2009 picoO $\mathrm{n}$, 2007). Rewriting code for more cores has a direct implication on production cos aer ma keting times and consumers end up paying for these shortfalls. In the ever incro $\mathrm{g} \mathrm{m}$ re processor cores the programmer has to rethink about the routines to $\mathrm{u}$ - and partitio $s$ the processing operations between the individual processors added.

3. 3. munications

Mul Ores present problems in the communication channels used by the processing elements to communicate between or to each other. PicoChip identified the "saturation of the communications links between processing elements" (Panesar et al., 2005, 2006; picoChip, 2007) as a major drawback especially to multi-cores with more than 10 processors. Race conditions are also "pernicious bugs" (Leiserson and Mirmam, 2008) that are difficult to detect. There is always need to have a reliable and efficient way to eliminate race conditions. Designing the interconnection channels between the various processing elements is crucial in order to achieve higher performance gains. The data or instructional dependencies may cause some of the processors to be idle hence loosing performance gains. The width of the communication 
channel is an important factor to consider. There is a concern that power dissipation can increase with multiple processing elements operating concurrently.

\section{4. Managing a heterogeneous architecture}

Multi-core systems are in most cases constituted by different types of processors and technically the architecture is referred to as a heterogeneous architectures. The heterogeneous architecture is not as easy to program as the homogeneous architecture that consist of similar processing elements. Homogenous architectures are easy to implement on silicon (picoChip, 2007, Hobson et al., 2006). Heterogeneous architectures provide greater yields in ion speeds because they include dedicated processing elements for specific applicati n tasks, su elements are designed to speed up code.

\section{5. Cache Memory Systems}

As mentioned earlier processor speeds have been scaling up ast resulting in the memory wall. Computer engineers have seen the botn pro sor a memory clock cycles have been decreasing overs time (processor by ab o \% per y Moore's Law and the memory by about $7 \%$ per year, Less' law) (Jesshop 2008, bson et al., 2006). There have been of course attempts to increase memory ban th by in wing concurrency in memory accesses through pipelining (Jesshope 2008 Hobson et al. 2, 06), but, this requires regular memory access patterns and random acce to the main memory bringing with it degradation in memory performance (Chevance, 200 Tesshope 2008 ). The memory hierarchy brings conflicting requirements in the memory systen. puting systems require a large and fast memory to scale up performances.

A memory hierarchy attempts to make a arge Memory appear fast by buffering data in smaller faster memories close to the proces (Heniessey and Patterson, 2007). Electronic systems slow down as they increa $\mathrm{ce}$ ze, for a ample the speed of light is approximately $1 \mathrm{~ns}$ for $30 \mathrm{cms}$ and $1 \mathrm{~ns}$ is 3 clock les ir a state o the art processor (Jesshope, 2008). Memory performance is therefore a mp power and performance, as is the processor performance today (Che ance, 200 Uennessey and Patterson, 2007). The key indicators of memory performance re memory ndwidth and latency (Hennessey and Patterson, 2007). Memory latency is dela ruired to obtain a specific item of data (measured in seconds), and, this is larg in dynamic $10 \mathrm{~m}$ access memory (DRAM) than in static random access memory (SB M) (I nessey and Patterson, 2007). SRAM can access any bit each cycle DRAM is res a to $b$ in the same row, cell address space (CAS) cycles. Memory Bandwi is the at which data can be accessed (e.g. bits per second), Bandwidth is nory ally, ycle th and this rate can be improved by concurrent access (Hennessey and

most common solution to the memory wall is to cache data and caching requires locality o cess or memory reuse, which may be achieved by compiler optimisations that can help to loc rise data (Jesshope, 2008). Computing scientists also designed banked memory systems to provide high bandwidth to random memory locations (Hennessey and Patterson, 2007; Jesshope, 2008), but, some access patterns still break the memory (Jesshope, 2008). Processors that tolerate high-latency memory accesses have been designed but this requires concurrency in instruction execution (Hennessey and Patterson, 2007; Jesshope, 2008). Caches are largely transparent to the programmer, but, programmers must be aware of the cache while designing code to ensure regular access patterns (Hennessey and Patterson, 2007; Jesshope, 2008, 2009, 2011). Caching the right data is the most critical aspect of caching to improve 
maximum system performances. More catch misses end up reducing performance instead of improving and this might end up consuming more memory and at the same time suffering from more cache misses lead to system deadlocks, where the data is not actually getting served from cache but is re-fetched from the original source. The development of a cache simulator requires a deeper understanding of how the memory hierarchy operates (Schintke, Simon, and Reinfield, 2012).

\section{DESIGN AND IMPLEMENTATION}

This research study is based on a simulating a 32KB 8-way set-associat Level1 D Cache. In this research study we have concentrated on the Shared Memoryarch ture. T] reason for choosing shared memory architecture is that we wanted to scale up ca ne simulator, from having one processor to a maximum of eight process $\mathrm{s}$ usin differe crace files. We have to modify the architecture to make sure that each pro ess de has cess to a local cache (reads and writes). The architectural implementation for this arch mplies that each processor node can write to a memory location, and it cache st o the memory contents locally, consequently a read of the same memory ation anothe processor node can be of a different value from its cache. The modifi ared men architecture used in this research is not unique as Jesshope (2011), sugge ed such memory architecture for scaling up processor frequencies. Associativity of caches (H and Smith, 1991) is an important metric that determine cache performance.

The implementation environment based on Systo 2005; Bhasker, 2009; Ma, 2011) resulted in lating a 32KB Level 1 data cache within the Arch Linux environment. We developed ste code for the implementation of the CPU, Memory, Cache, Bus and used Iesshope 2011) Trace Files used to drive the simulator. For our simulation we used the $\triangle N_{\mathbf{M}}$ platfor Arch Linux 3.8 (http://www.archlinux.org) with GNU C++ compiler ve $\mathrm{g}$ g-4.8. It s one of the lightweight GNU/Linux based

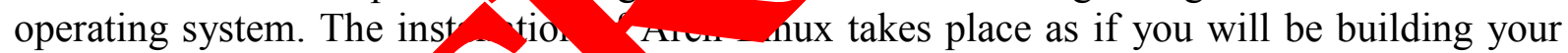
own operating system, o is heavl, ommand driven. The three main issues that one should take care of when ins alli 4 rch Linu is the graphics, network especially wireless networks and UEFI. We cho insta because of havig used it in and Linux ambience which is the Linux Mint environment. We followed the nstruc ns in the INSTALL document that comes with the SystemC-2.2.0 package to con it in Ay Linux. The SystemC installation is a nasty experience and it took us som to cu ile and run in Arch Linux. Jesshope (2011) provided the theoretical and pro amm g found ons of the trace files used for this research, and we use his trace files vers 3. J hic nhlosophy behind these trace files to drive our simulator.

approach to implement the SystemC Level 1 Data-cache simulator followed the conventio programming norms of increasing the programming complexity as the demands of the syste $n$ increases. We started by implementing a bus snooping cache coherence protocol, the Valid-Invalid protocol. The term 'snooping' allows for each cache node in the system to monitor the activities on the bus to which each of the cache nodes can write exclusively. In the event of a write enquiry if a cache node realizes that another processor belonging to another cache node has written to an address which it has a copy, the cache line containing a stale copy of the associated memory segment is immediately invalidated. The programming logic behind this protocol is that it does not allow for two cache lines to be valid in different cache nodes, in the event that they are mapped into the same set and even share the same address tag. The 
implementation of this protocol served as the basis for diagnosing anticipated programming problems and we used the debugging traces to eliminate errors until we were satisfied with the program executions.

We then implemented the MOESI Cache coherence protocol which is theoretically and programmatically built as an extension to the MESI protocol. The MESI protocol is the most common cache protocol that supports the write-back replacement strategy. The acronym MESI indicates that the protocol supports four cache line state transitions and these are Modified, Exclusive, Shared and Invalid, which logically implies that it implements the same cache line invalidation scheme as the valid-invalid cache coherency protocol. The difference to thovalidinvalid cache coherency protocol is that it monitors whether the cache line is share or no caches are allowed to make the cache line dirty if the cache line is in a modif or exclus state. The MOESI cache coherence protocol introduces a fifth cache line tra ition sta 'owned' which means it has characteristics of exclusive modified and s'ared cach o s'ate transitions. We have to point out that this cache coherency protocol all vs for she lin to be shared, and is not supposedly written back to memory before the sh rint

As a starting point we build a single $32 \mathrm{~KB} 8$-way set asso ative cac vith 2 Byte line size. We also built a CPU module connected to the cache that ving for 1 ing or writing some data from or to memory through the cache. In additi we a memory module to help in checking the correctness of the data. The connec has been made from an 8-bit wire, therefore to fill the 32 Byte cache lin $\iota$, the cache has to read the memory 32 times. This was also useful to simul the memory latency. We only used the random trace file for one processor to test the corre ess of ou simulator. The result of the simulation can be seen in the Table 1.

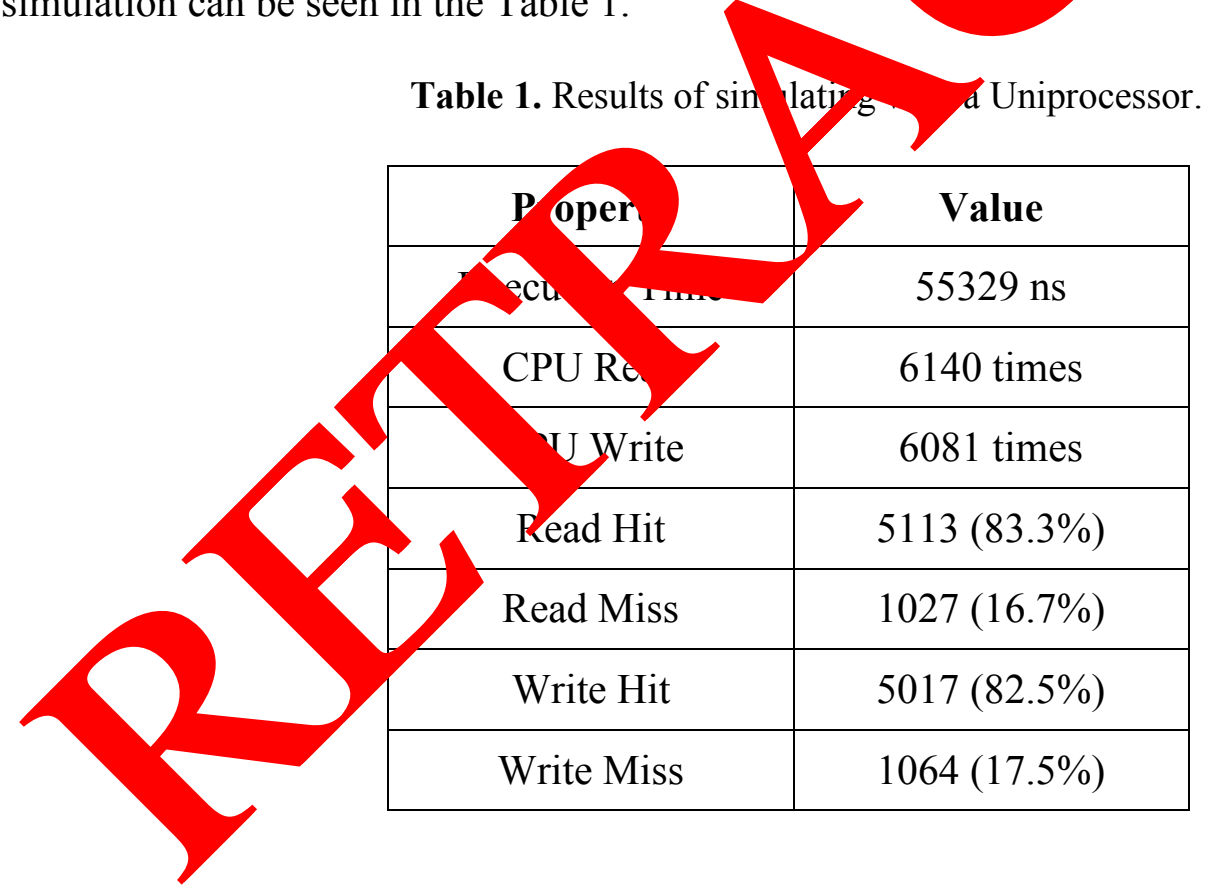

The results in the show that the CPU made 12221 requests composed as 6140 read requests and 6081 write requests. The results further show that more than $80 \%$ of the requests hit the cache, with an execution time of $55329 \mathrm{~ns}$.

\section{1. Comparative Results Using Graphs}

We plotted graphs to make a fair comparison of the trace files used and also the snooping and directory based cache coherency protocols. We made a comparative analysis of the 
protocols considering that there is no bus snooping, no barrier synchronization and with barrier synchronisation for each protocol. We started by comparing the Average Cache hit Rate and the two graphs represented by Figure 1 and Figure 2 indicate that there is no major significant difference between the Valid-Invalid and MOESI cache coherence protocols in terms of the cache hit rates, when random trace files are used. The different configurations made to the simulator did not show distinguishable cache performance indicators between the two sets of traces. The MOESI protocol theoretically outperforms the Valid-Invalid protocol.

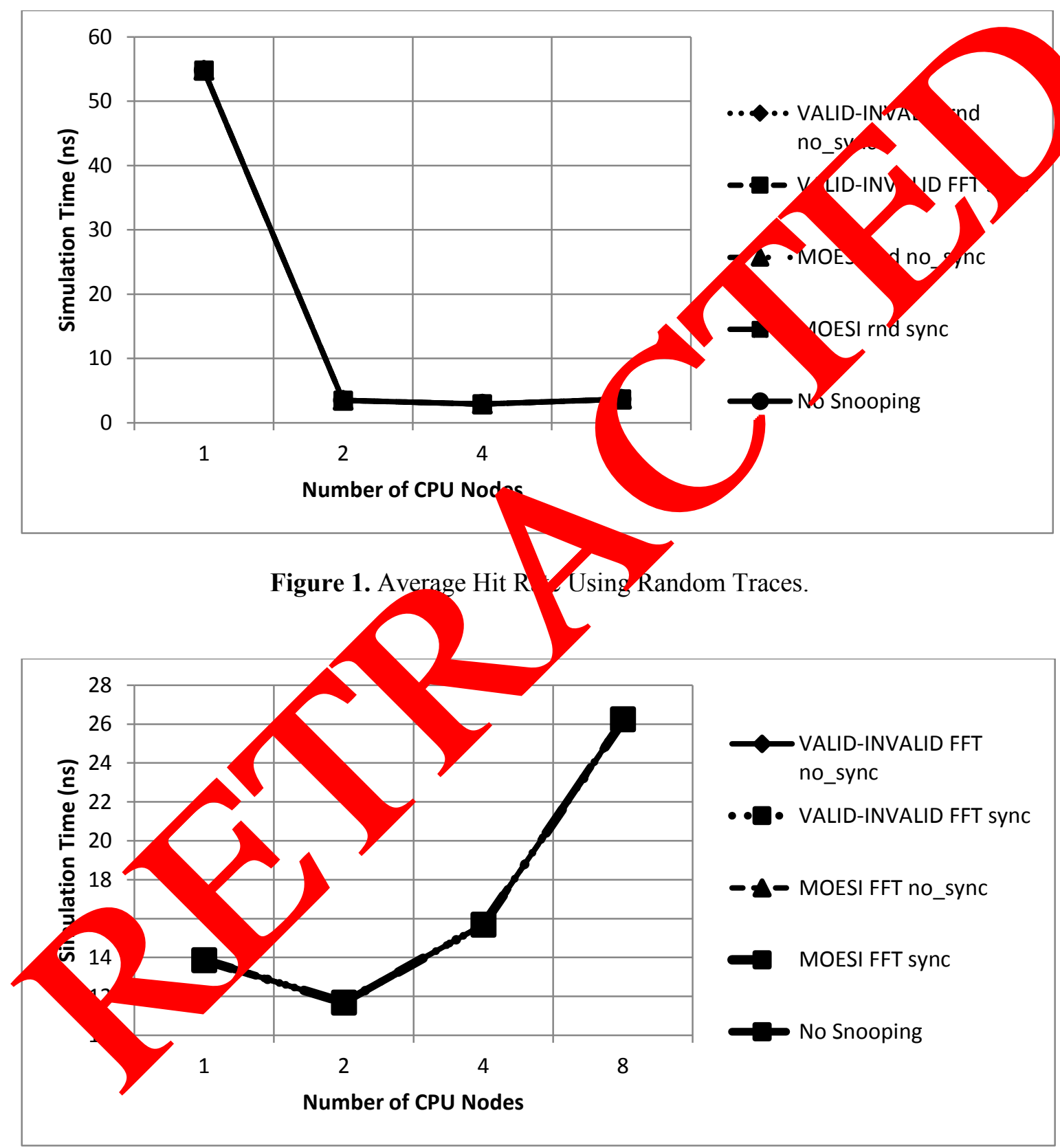

Figure 2. Average Hit Rate Using Fast-Fourier Transform Traces.

The other result that was very important to the SystemC cache Simulator experiment was to investigate the contention of the bus interconnection network. This was achieved by taking a count of the time stamps (delta cycles) in which the bus had more than one request to handle. This was handled by a member function in the Bus module which was designed to indicate the 
number of requests in the queue. The bus contention when using the two sets of traces is shown by Figure 3 and Figure 4.

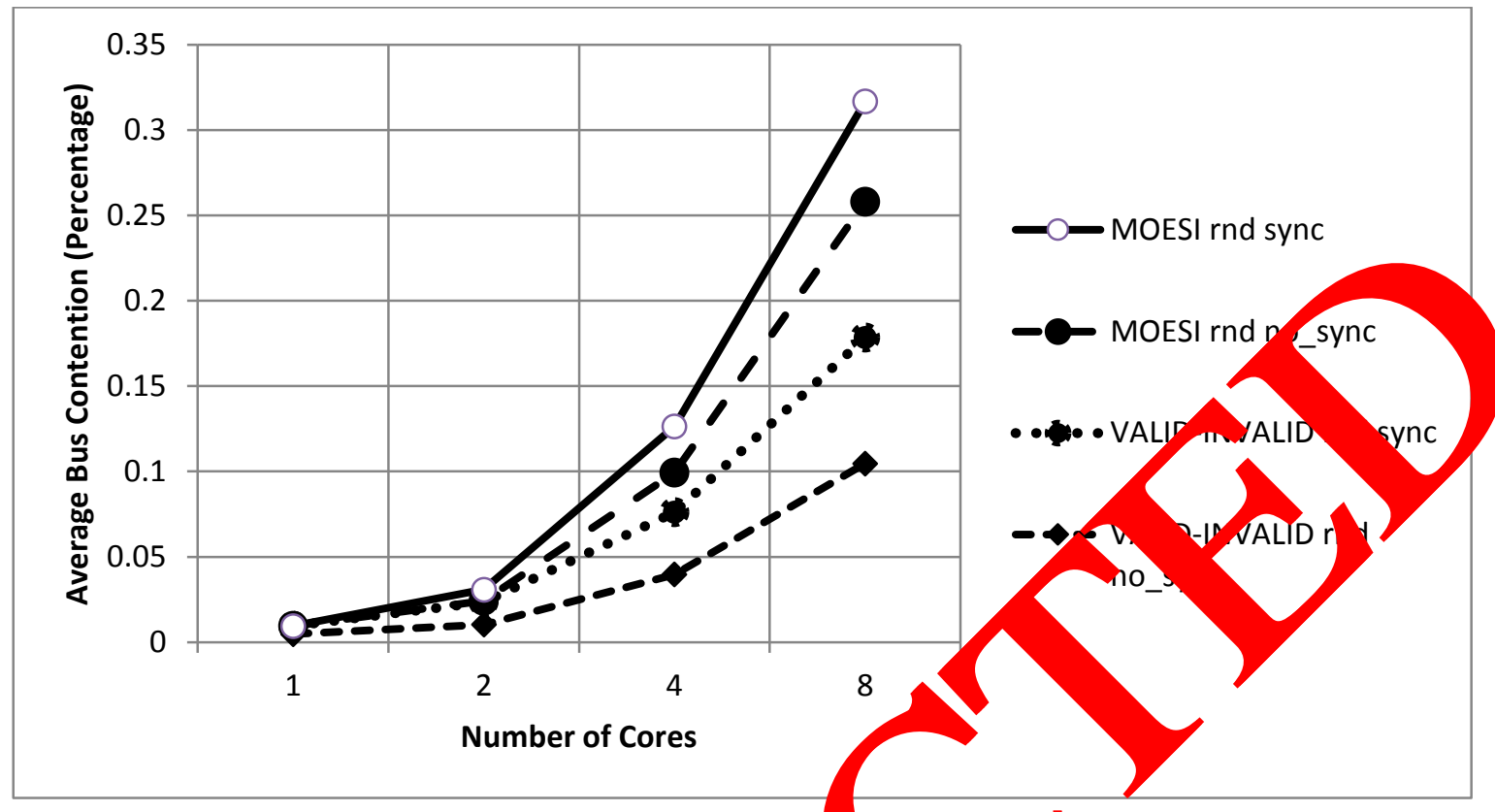

Figure 3. Average Bus Contention Using -Fourier T ansform Traces.

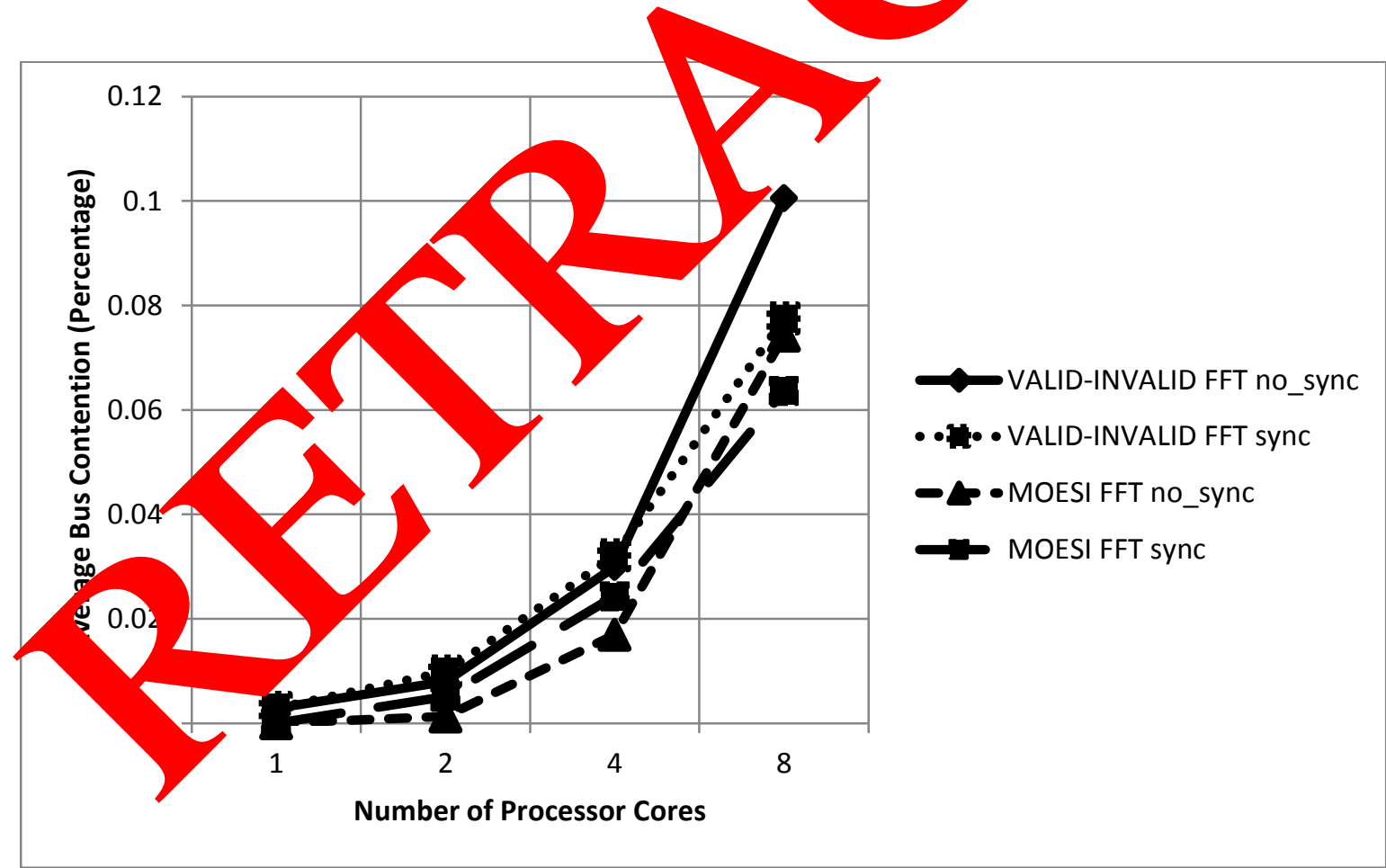

Figure 4. Average Bus Contention Using Fast-Fourier Transform Traces.

The synchronised cache simulator runs show a reduction in the bus contention. The synchronisation event relieves the interconnection network as it oblige the processor nodes to wait until the barrier threshold instead of putting them in a race condition towards the end of 
each trace. The MOESI cache coherence protocol exhibit a smaller footprint on the interconnection network (bus), due to the deferred writes, but consequently uses more memory resources.

\section{CONCLUSIONS}

The SystemC cache simulator we have developed initially showed some feeble plugs, maybe because, of the fact that the trace files we have used in the simulation were designed to pick up read and write addresses for (hits/misses), instead of showing how the data ic moved around in the system. In that way we would actually have testified that th proco s constituted in the system have actually performed the reads and writes of the ta they w supposed to. We also noticed that even if the trace files provided for cherking hether $t$ processors $\mathrm{read} / \mathrm{write}$ the data they are supposed to, there is no assy ance that ca ne simulator is correct. We introduced a component of non-determinis in thent at the different cache nodes attempted simultaneously to access the bus.

The introduction of a memory latency of a century of cycle did not g vally ssume that a read issued just a few cycles after a write onto the same me $n$ address, 1 harvest the correct data response. If the memory was responding to a rea requ vithin the memory cycle latency limit, a write request issued to the same memors auress was permible, and the stale data value was not send back to the bus. The cr the coherency procols resolved such a situation by implementing two further cache cohere y organizatipns, and these are the writeinvalidate or the write-update.

As Jesshope (2011) argued that write-invalida the management of dynamic requests and the logic to rearrange reques hardware in the form of a buffer that will ont $1 \mathrm{~m}$ addresses of the requests, and the associated data elements, forcing the main $n$ mory to behave the same as the cache. We implemented the write-invalidate sche $\mathrm{e}$ as it is conservative and compatible with our chosen cache coherency protocols. It her y uned aw 4 the existence of duplicate read requests by allowing for a small degre pe stimizations. We studied the graphs and come to the conclusion that cac coherenc otocols are comparable, even when we use different traces and different $\mathrm{p}$ mb f process $\mathrm{s}$. We therefore use the experimental data and graphs to answer our rese ch quest.

\section{Answering th Rese rch Ques, ons}

The first rch que tion refers to an investigation of the performance of the cache when we incr the $\mathrm{n}$ her $\mathrm{g}$ processors. Based on this postulate we then give our response to the follo wing st resea question entitled:

To at and do the number of processors in multiprocessor architectures affect the perfon "ce of Level-1 (L1) data cache memory systems?

Wo renoted that the runs of all the cache simulator experiments we have made did not end up in inconsistent state. The execution time (simulation time) of the cache simulator increases as we have more processor cores. The average hit rate did not increase significantly with the increase of the processor cores. We have also noted that other factors such as snooping have a direct effect on the performance of the cache. From the results of the simulations we could see that increasing the number of cores does not imply an increase in cache performance as there are coherency issues to be taken care of. The deactivation of the snooping on the interconnection network subsequently increased the average hit rate even when using different trace files. 
Without snooping on the bus, there is now invalidation in case of probe write hits, meaning that the cache writes to a shared cache line and the status of the cache line remains the same. In such an instance the cache gets a higher hit rate. As performance is determined by the hit rate we would argue that the cache performs much better without snooping. However when we deactivated bus snooping we could not guarantee and assure the integrity of the cache line when we repeatedly run the cache simulator. The other factor that comes into play when we increased the processor nodes is synchronisation of the caches and taking care of the cache misses. One way of taking care of this aspect is to optimize the compiler, by code rearrangement including data rearrangement. Loop interchange and cache blocking could also optimiae the cache by improving temporal locality. We can conclude that increasing the numb of processors on the multiprocessor architecture implies more cache programming mplexity a cache coherency is a major concern in the performance of the caches of a $\mathrm{m}$ iprocess system.

Rightfully we can say that given optimizations in the compiler o havi synch sised multibanked caches in the multiprocessor system, we can increase perfor ance. As mentioned earlier increasing processor nodes with their local car es mean the $c$ is a lot of programming issues to consider. In our case we pipelined th a access at we would increase the cache bandwidth. We have mentioned earlier th cach herency is an important aspect to consider in a multiprocessor environment. We arc fore invo roted how our chosen cache coherency protocols affected the performang of our cache sy nulator. The research question to answer is the folloing:

How do cache coherency protocols influence th vel-1 1) data cache memory performances of mu acessor annectures?

We have used trace caches to reduce th hit an system henceforth improve the cache hit rate. Each implementation of our Syst ${ }_{1} \mathrm{C}$ cacne simulator had to run a set of Random and Fast-Fourier Transform trace nTes 1, 2, 4, nd 8 processor environments. The comparison graphs showed that the dire $\mathrm{v}-\mathrm{ba}$ ed cache coherence protocol (MOESI) has a slight performance edge over the noo onence protocol (Valid-Invalid). Though the difference can be regard as statis 1ly, insignificant, MOESI protocol outperforms ValidInvalid protocol beca se n transfer ata from one cache to another cache. In such cases the cache miss doesn' a yays in the cache has to read/write from/to memory. Lesser memory access reads lea $\mathrm{s}$ to faster exe on time because the need to wait for memory access latency can be reduc The ratio of he MOESI protocol is better than the hit ratio in Valid-Invalid protocol mean at cons cutive writes will always contribute to a cache miss. In the MOESI protoco writ iss o curs, the cache line will be updated (read) and the consecutive write will e ma ed as w nit. Another contributing factor to the better performance of the MOESI pro. is hot it hag a lower contention rate of the bus usage. One of the reasons for this could be tha e menrory access rate in Valid-Invalid protocol is more than in the MOESI protocol. Since tho cywill be used when the cache modules want to have memory access, higher memory ac ess will imply a higher request to use the bus. Following the memory hierarchy principles, accessing the bulk shared memory will take more time compared to accessing another cache. The Valid-Invalid have to wait longer to access the memory than in MOESI protocol.

Unexpectedly in some instances the MOESI cache coherence protocol used more memory writes which might be as a result of a bug in our SystemC cache Simulator. We have actually managed to preserve the coherency of the caches in all our experiments and all simulations. We still need to conduct a proof of the program correctness of our simulator using acceptable, 
scientific, standard proof-of-program correctness methodologies. All the simulations never ended up in an inconsistent state, which is a significant leap towards the optimization of the cache simulator. We therefore have the following recommendations for the improvements of the cache simulator.

\section{RECOMMENDATIONS}

The performance graphs showed that there is no significant performance diffarence between the snooping protocol and the directory-based protocols we have chosen. Tieore this is wrong and one of the reasons is that there might be a programming erro bug) in bookkeeping of the memory writes through the traces used or in the cache simula itself. $V$ therefore recommend a program proof-of-correctness procedure to be co iried out a alse to revise the configurations of the trace files. The Valid-Invalid protocol $g$ perfor $d$ the JESI protocol when random trace files were used which is a point of $\mathrm{nco}$ ne cac $\mathrm{s}$ cannot expect randomness as they are based on programming attributes and the co rency attribute is a result of programming efforts. We therefore recommend a it on the files and an increase in the range including the types of trace files to be ed by simulator.

We have not taken into consideration issues of asing the bandwidth. As a future area of research and improving the cache perfo nance we have to consider various cache optimizations schemes and also record the data for t memory arcesses. The implementation of various cache optimizations will bring an incre in progr $m$ complexity of the cache simulator. Concurrency has been a major nrogramm during the execution of the simulator. When we implemented the Syste mulator we had Error (115), which did not allow us to start the simulator with two or $m$ re have actually resolved this error by implementing SC_SIGNAL_WRITE_CH CK= "DISABLED" at the start of each simulation involving more than one cessor ut we recommend that we have to create an environment variable that alle for e slicit par ilelism to occur during the simulation.

We also recommend us a r ange of cache coherency protocols rather than choosing only one type ach categ As SystemC can be implemented in the multi-platform environment and the mu exhibits ne characteristics of the hardware being simulated, we will in the try the mulator ferent multiprocessor environments. However this has been a learning curve for us and this ro ich is useful in multiprocessor design.

\section{Refer}

[1] to . Ginfri R., Journal of Embedded Computing 2 (2003) 137-139.

[2] Bha J. (2009). The SystemC ${ }^{T M}$ Primer. Allentown, Star Galaxy Publishers.

[3] Black 9., D., Donovan J (2004). System C from the Ground Up, Boston, Kluwer Publishers.

[4] Byler J. (2009). Software Programmers face Multicore Challenges, in Embedded Intel. [Online]. Intel. Available: http://www.embeddedintel.com/from_intel.php?article=1050 [Accessed 11 March 2013 2013].

[5] Chevance J. R. (2006). Servers Architectures: Multiple Processors, Clusters, Parallel Systems, Web Servers, Storage Solutions, London, Elsevier. 
[6] Duller A. P. G., Towner D. (2003). Parallel Processing - the picoChip way! . In: Broenik, J., F And Hilderink, G. (ed.) Communicating Process Architectures. London: PicoChip.

[7] Hennessy L. J., Patterson A. D (2007). Computer Architecture. A Quantitative Approach, San Francisco, Morgan Kaufmann.

[8] Hill D., Smith A. J., IEEE Transactions on Computers 40 (1991) 371-390.

[9] Hill D., Smith A. J., IEEE Transactions on Computers 38 (1991) 1612-1630.

[10] Hobson R., Cheung K. L., Ressi B.,Signal Processing with Teams of Embedded Workhorse Processors. EURASIP Journal on Embedded Systems (2006) 16.

[11] Jesshope C. R. (2008). A model for the design and programming of mult res. In: GRANDINETTI, L. (ed.) Advances in Parallel Computing, 16, High rror Computing and Grids in Action London: IOS Press.

[12] Jesshope C. R (2009). Multiprocessor Memory Systems, Amstera Ur versit it ian Amsterdam

[13] Jesshope C. R .(2011). A SystemC Tutorial. Amsterdam

[14] Joubert G. R. (2008). "Parallel computing current of high end computing", Forschungszentrum, John von Neu ann lnstitute fo, omputing Jülich

[15] Leiserson C. E, Mirman I. B. (2008). How to S vive the Sur ive Multicore Software Revolution [or at Least Survive the Hype]. . In. ICKART's, I. (ed.). New York: Click Arts Inc.

[16] Ma N., "Modelling and evaluation of $m$ tumultithreaded processor architectures in SystemC", Proquest, (2011) 1109.

[17] Mckee A. S. (2004). Reflect the Mery Wall. In: ACM, ed. Proceedings of the 1st conference on Comp $\mathrm{g}$ fro tiers (CF J4). 2004 New York. New York: ACM, 1-6.

[18] Nussbaum S., Smith (2) Staun Cal Simulation of Symmetric Multiprocessor Systems. In: IEEF In Proce of the 35th Annual Simulation Symposium (SS '02) , 2002. W hin DC, U\$ A, 89. IEEE Computer Society, 89-97.

[19] OSCI. (200 ). An Introdu to System Level Modelling in System C 2.0. Available: www.es tle.tue $\mathrm{v} /$ heco/courses/EmbSystems/WhitePaper20.pdf [Access 15/arch 013].

[20] P r G. Daler A., Gray A., Robins W. (2005). Deterministic Parallel Proc sing. In. KOENIK, J., F AND HILDERINK, G. (ed.) Microgrid workshop0,5 .

[21] Pà ar G. T. D., Duller A., Gray A., Robins W., International Journal of Parallel Prog, mming 34(4) (2006) 323-341.

[22] PICOCHIP. (2007). Technical White Paper: Practical, Programmable Multi-Core DSP. Available:

http://www.picochip.com/downloads/4eac6c97aa70840ad7f4d12aec82ebf1/Multicore_J une_2007.pdf [Accessed 13 March 2013].

[23] Schintke F., Simon J., Reinfield A. (2012). A Cache Simulator for Shared Memory Systems (unpublished paper)

[24] Stalling W. (2012) Computer Organization and Architecture London, Prentice Hall. 
[25] Sutter H. 2005. The free lunch is over: A fundamental turn toward concurrency in software. [Online]. New York. Available:

http://www.gotw.ca/publications/concurrency-ddj.htm [Accessed 13 March 2013 2013].

[26] Szydlowski C. (2005). Multithreaded Technology \& Multicore Processors [Online]. New York: Dr. Dobb's. Available:

http://www.drdobbs.com/multithreaded-technology-multicore.../18440607 [Accessed 13 March 2013 2013].

[27] Towner D. P. G., Duller A., Gray A., Robins W. (2004). Debugging and Ver jicalıo Parallel Systems - the picoChip way! In: BROENIK, J., F AND HILDER IK, G. (ed. Communicating Process Architectures London: IOS Press.

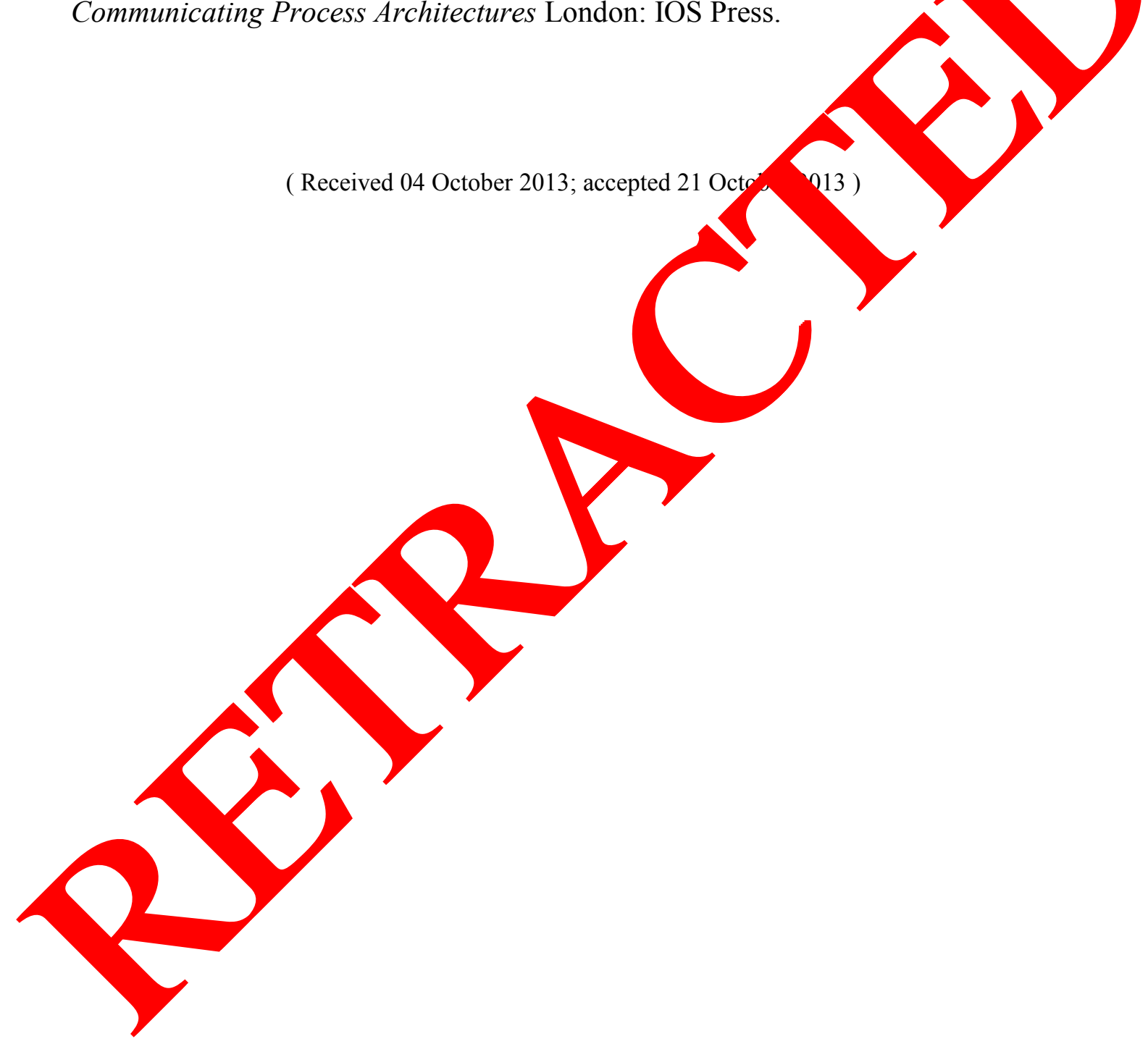

